\title{
Maternal systemic lupus erythematosus and chrondrodysplasia punctata in two sibs: phenocopy or coincidence?
}

\author{
Nursel Elçioglu, Christine M Hall
}

\begin{abstract}
Two sibs with chondrodysplasia punctata in whom the mother was suffering from systemic lupus erythematosus are presented and the radiological features described. Comparison with other forms of chondrodysplasia punctata with a review of the relevant publications is presented and the possible asscociation with maternal systemic lupus erythematosus is highlighted.

(F Med Genet 1998;35:690-694)
\end{abstract}

Keywords: systemic lupus erythematosus; chondrodysplasia punctata; metacarpal type; brachytelephalangic type

Chondrodysplasia punctata (CDP) is an extremely rare inherited disease which has several described forms. It is characterised by a distinctive radiographic appearance of localised stippling particularly in the spine and the region of the epiphyses. It is observed prenatally and in infancy but disappears during childhood. ${ }^{1}$ Stippling is also seen in a heterogeneous group of disorders, including some teratogenic exposures. It has been reported following maternal exposure to warfarin, dilantin, phenytoin, and alcohol as well as rubella embryopathy and deficiency of vitamin $\mathrm{K}$ caused by embryonic enzyme deficiency or maternal malabsorption or malnutrition..$^{1-4} \mathrm{~A}$ direct association with a maternal autoimmune disease such as systemic lupus erythematosus (SLE) has not been reported previously.

\section{Case report}

The mother, a 29 year old black African sustained two intrauterine deaths, one at 36 weeks of gestation the other at 24 weeks. Her healthy black African partner was not consanguineous. She was suffering from SLE and had intermittant steroids during both pregnancies. These intrauterine deaths represented consecutive pregnancies. There had been previous spontaneous miscarriages but no details were avaliable.

\section{CASE 1}

This was a male stillbirth of 36 weeks' gestation. The maternal SLE had been active in the past but at the time of conception was quiescent with only a persistently painful right hand and wrist. She was taking prednisolone $15 \mathrm{mg}$ daily both before and during pregnancy but no other medication. Ultrasound scanning at 22 weeks showed numerous fetal abnormali- ties including nasal hypoplasia, short long bones, and a sacral abnormality. Polyhydramnios was present. Karyotyping showed normal male chromosomes, 46,XY. The couple elected to continue the pregnancy. Repeat scanning showed parallel growth with femoral lengths well below the 3 rd centile, head circumference on the 20th centile, and abdominal circumference between the 3 rd and 10 th centiles. A scan at 36 weeks' gestation showed no fetal heart activity and intrauterine death was diagnosed. Induced labour resulted in a stillborn male.

Gross inspection at necropsy identified a macerated male infant, weighing $1485 \mathrm{~g}(<5$ th centile), length $27.5 \mathrm{~cm}$ ( $<5$ th centile), head circumference $31 \mathrm{~cm}$ (10th centile), and right foot length $49 \mathrm{~mm}$ (corresponding to 26 weeks' gestation). There was mild generalised oedema. Dysmorphic features included a poorly developed bridge of the nose, with resultant absent nasal contour, but normally formed nostrils, and no cleft lip or palate. There was symmetrical, mild limb shortening. The shape and position of the digits was abnormal with short fingers and camptodactyly. The toes overlapped and there were poorly formed hypoplastic nails, particularly on the feet.

Radiology showed (fig 1) soft tissue oedema and short long bones with pronounced humeral shortening. All long bone measurements were below the 5 th centile for the chronological gestational age. Femora (55 mm), tibiae (49 $\mathrm{mm}$ ), and radii ( $43 \mathrm{~mm}$ ) were on the 50th centile for 29 weeks' gestation, but humeri (43 $\mathrm{mm}$ ) were even shorter, below the 5 th centile for this gestation.

The skull vault was dolichocephalic and poorly ossified. There was quite striking hypoplasia of the facial bones resulting in midface hypoplasia. The laryngeal cartilage showed premature calcification. The sternal centres were deficient There was stippling throughout the length of the spine anterior to the vertebral bodies in the region of the anterior spinal ligament. A little stippling was present more laterally overlying the region of the pedicles. There was deficient ossification of the anterior parts of the vertebral bodies making identification of coronal clefts difficult. Several upper thoracic vertebral bodies showed sagittal clefting. There was some shortening of long bones, particularly the humeri, without metaphyseal flaring. There was stippling at the proximal femora but no stippling in the region of the knees (fig 1A, B). Minimal stippling was seen in the region of the elbows and at the proximal ends of the 
short 1st, 2nd, 3rd, and 4th metacarpals (fig $1 \mathrm{C})$. In the feet, stippling affected the region of the tarsal bones and the proximal ends of the 1 st, 2nd, and 3rd metatarsals (fig 1D).

CASE 2

This 24 weeks' gestation fetus was the sib of case 1 and the second affected pregnancy. This fetus was lost during a maternal flare up of SLE. During the pregnancy, in addition to prednisolone and paracetamol, the mother was
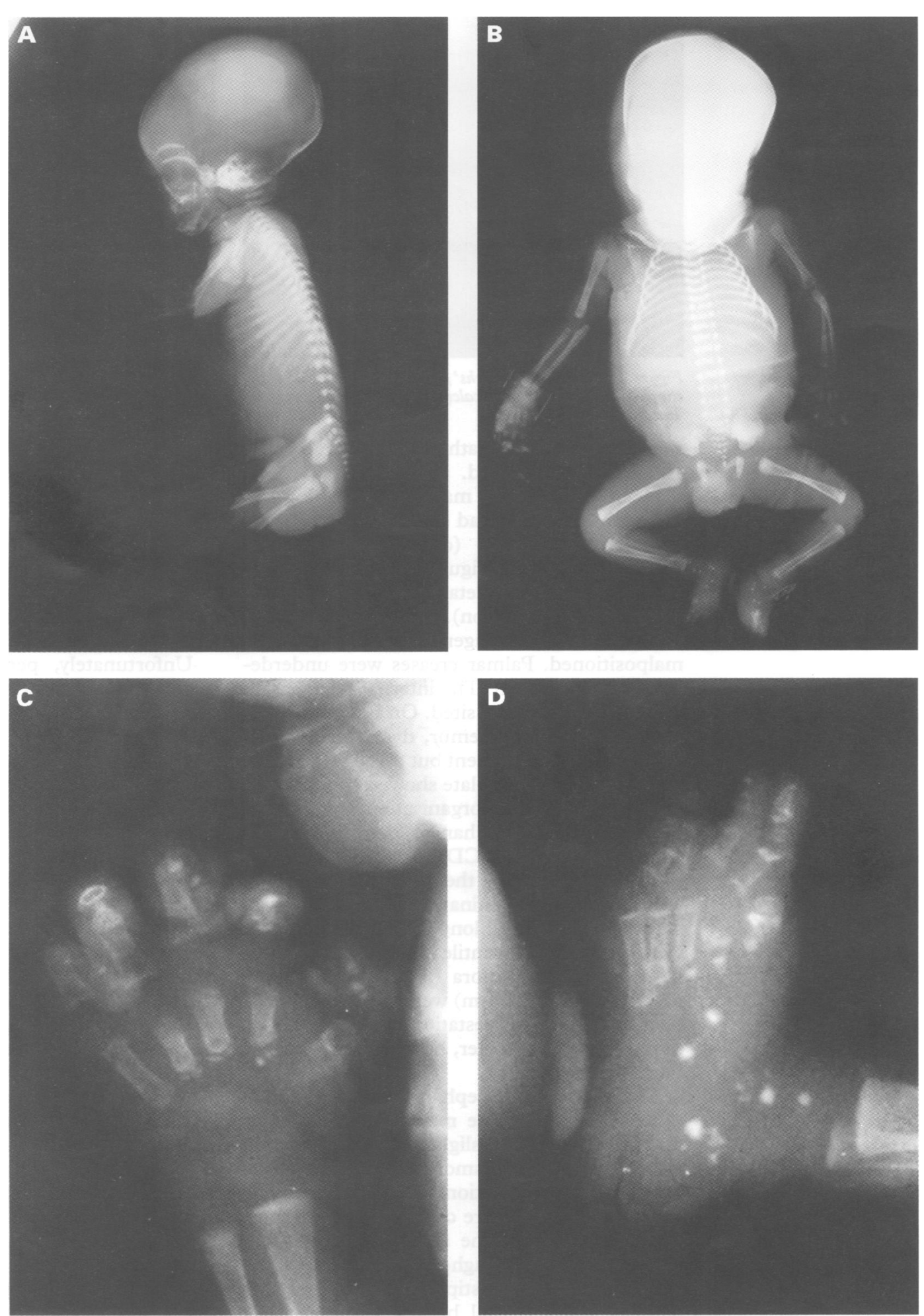

given aspirin $75 \mathrm{mg} /$ daily. In the early part of pregnancy, fetal growth was normal but by 20 weeks the biparietal diameter failed to increase. Femoral length was below the 3 rd centile from week 19 onwards. At 21 weeks maternal urine protein excretion increased to nearly $5 \mathrm{~g}$ in 24 hours. By 23 weeks the mother had florid SLE requiring increased steroids. There were complications of renal failure requiring dialysis, malignant hypertension, and severe neurological impairment. At 24

Figure 1 Case 1 at 36 weeks'gestation. (A) Lateral babygram. Midface hypoplasia. Stippling in the region of the anterior vertebral bodies. Calcified laryngeal cartilages. (B) Anteroposterior babygram. Stippling of proximal femora and sacrum. (C) Posteroanterior left hand. Short 1st-4th metacarpals with proximal stippling. (D) Lateral foot. Tarsal stippling. Short 1 st, 2 nd, and 3rd metatarsals with proximal stippling. 

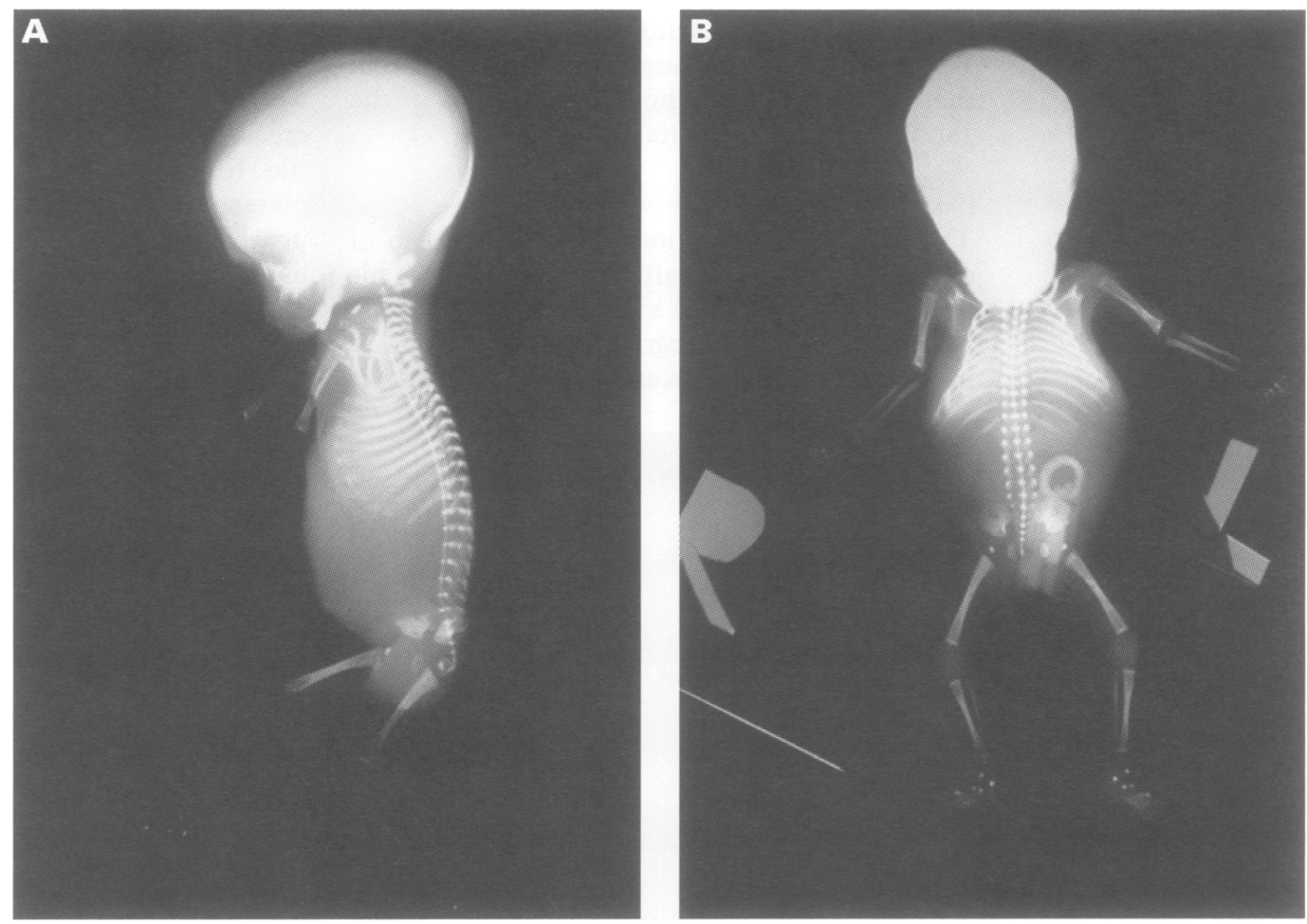

Figure 2 Case 2 at 24 weeks'gestation. (A) Lateral babygram. Continuous ossification of anterior spinal ligament with stippled laryngeal cartilage calcification. (B) Anteroposterior babygram. Stippling of proximal femora and distal humeri. Tarsal stippling.

weeks intrauterine death was confirmed and a stillbirth was delivered.

Necropsy showed a male fetus weighing 190 g, length $20.7 \mathrm{~cm}$, head circumference $16 \mathrm{~cm}$, foot length $25 \mathrm{~mm}$ (corresponding to 18 weeks) and these figures indicated severe intrauterine growth retardation $(<5$ th centile for 24 weeks' gestation). The limbs appeared slightly short. The fingers appeared short and malpositioned. Palmar creases were underdeveloped on the right. The internal organs were correctly formed and sited. On bone histology of spine and upper femur, dystrophic foci of calcification were present but ossification at the metaphyseal growth plate showed chondrocyte columns with no disorganisation and normal ossification. These changes were consistent with the diagnosis of CDP, but did not help to differentiate between the different aetiologies.

Radiological examination (fig 2) showed short long bones. All long bone measurements were below the 5 th centile for the chronological gestational age. Femora $(27 \mathrm{~mm})$, tibiae (23 $\mathrm{mm})$, and radii $(23 \mathrm{~mm}$ ) were on the 50 th centile for 18 weeks' gestation but humeri (21 $\mathrm{mm}$ ) were even shorter, below the 5 th centile for this gestation.

There was dolichocephaly and poor ossification of the vault. The mandible was straight and short. There was slight midface hypoplasia although no facial dysmorphism was noted on postmortem examination. The laryngeal cartilage showed premature calcification. Continuous calcification of the anterior spinous ligament was noted throughout its length and this was associated with stippling in the anterior parts of the vertebral bodies. Coronal clefts were present (fig 2A). There was a little stippling at the distal end of the humeri and this appeared to be incorporated into the

metaphyseal region. Stippling was also present at the proximal ends of the femora. There was no stippling in the region of the knees. In the feet, stippling was present in the region of the tarsus and the 1st metatarsals were short. In the hands, there was significant shortening of the $3 \mathrm{rd}$ and 4 th metacarpals although at this stage no stippling could be identified in these areas (fig 2B).

Unfortunately, peroxisomal and chromosomal studies could not be performed in our two cases.

\section{Discussion}

Both cases show features of chondrodysplasia punctata. There is stippling in the tarsal and upper femoral regions and anteriorly along the spine, especially in the 24 week fetus with ossification of the anterior spinal ligament and coronal clefting. There is also ossification in the laryngeal cartilages. There is evidence of symmetrical long bone shortening, shortening of the metacarpals and of several phalanges, especially the proximal phalanges of the index fingers and the terminal phalanges. In case 1 , stippling was more pronounced but this could be related to the older gestational age. There was no stippling at the knees although this is commonly seen in many forms of CDP. Both cases had midfacial hypoplasia. In both pregnancies intrauterine death may have resulted from severe maternal SLE and hypertension since, in general, in all forms of CDP intrauterine death is unusual.

Although many types of CDP are recognised, our cases correspond most closely to CDP metacarpal type and brachytelephalangic $\mathrm{X}$ linked recessive type. Distinguishing between these may be difficult. In the metacarpal 
Table 1 Chondrodysplasia punctata cases associated with maternal systemic lupus erythematosis

\begin{tabular}{|c|c|c|c|c|c|}
\hline \multirow[b]{2}{*}{ Features } & \multicolumn{2}{|c|}{ Costa et al ${ }^{13}$} & \multirow[b]{2}{*}{ Mansour et al ${ }^{\prime \prime}$} & \multicolumn{2}{|l|}{ This report } \\
\hline & Case 1 & Case 2 & & Case 1 & Case 2 \\
\hline $\begin{array}{l}\text { Sex } \\
\text { Gestation (wk) } \\
\text { Birth weight }(\mathrm{g}) \\
\text { Dolichocephaly and poor ossification of the skull vault } \\
\text { Midface hypoplasia } \\
\text { Brachydactyly/short MC/MT } \\
\text { Vertebral clefting }\end{array}$ & $\begin{array}{l}F \\
29\end{array}$ & $\begin{array}{l}M \\
36\end{array}$ & $\begin{array}{l}M \\
25 \\
920 \\
+ \\
+ \\
+ \\
\text { Sagittal and coronal }\end{array}$ & $\begin{array}{l}\text { M } \\
36 \\
1540 \\
+ \\
+ \\
+ \\
\text { Sagittal and ?coronal }\end{array}$ & $\begin{array}{l}\text { M } \\
24 \\
190 \\
+ \\
+/- \\
+ \\
\text { Coronal }\end{array}$ \\
\hline Localisation of $C D P$ & $\begin{array}{l}\text { Hips and } \\
\text { tarsus }\end{array}$ & $\begin{array}{l}\text { Hips and } \\
\text { shoulder }\end{array}$ & Metacarpals and spine & Metacarpals, metatarsals & arsus, spine, hip, elbow \\
\hline $\begin{array}{l}\text { Laryngeal cartilage } \\
\text { Spine } \\
\text { Hips } \\
\text { Knees } \\
\text { Tarsus } \\
\text { Shoulder } \\
\text { Elbow } \\
\text { Metacarpals } \\
\text { Treatment during pregnancy for maternal SLE } \\
\text { Outcome } \\
\text { Origin }\end{array}$ & $\stackrel{+}{\text { Alive } 1 \mathrm{y}}$ & $\begin{array}{l}+ \\
- \\
+ \\
+ \\
+ \\
\text { Died } 6 \mathrm{mth}\end{array}$ & $\begin{array}{l}- \\
+ \\
- \\
- \\
+ \\
- \\
+ \\
+ \\
\overline{+} \\
\text { Alive }>5 \text { y } \\
\text { AfroCaribbean }\end{array}$ & $\begin{array}{l}+ \\
++ \text { ant ligament } \\
+ \\
- \\
+ \\
- \\
+ \\
+ \\
+ \\
\text { Stillbirth } \\
\text { African }\end{array}$ & $\begin{array}{l}+ \\
++ \text { ant ligament } \\
+ \\
- \\
+ \\
- \\
+ \\
+ \\
+ \\
\text { Fetal loss } \\
\text { African }\end{array}$ \\
\hline
\end{tabular}

type, in addition to short metacarpals, usually involving the $3 \mathrm{rd}$ and 4 th metacarpals, there may be shortening of the proximal phalanges of the index fingers with some radial deviation of these fingers. ${ }^{1}$ In addition, there is long bone shortening which has been reported as predominantly affecting specific limb segments in various series. Major tibial involvement is reported in the series of Ritter et al and Argo et $a l^{6}{ }^{6}$ mesomelic shortening by Burck, ${ }^{7}$ and humeral shortening by Borochowitz, ${ }^{8}$ but the long bone shortening may be generalised. Typically, the stippling is mild affecting the lower spine, carpals, and tarsals and there are coronal clefts. Clinically there is severe midface hypoplasia, normal psychomotor development, and no ichthyosis or cataract formation.'

The brachytelephalangic type has $\mathrm{X}$ linked recessive inheritance and is seen only in males. The distal phalanges are short with a triangular appearance, being wider distally, but in the older child they appear short with a relatively wide epiphysis at the proximal ends of these phalanges. Many reported cases have also shown short metacarpals and some also short proximal phalanges of the index fingers causing radial deviation. ${ }^{9-12}$ Long bone shortening is not a feature. The facial dysmorphology, absence of cataracts, and ichthyosis with a good prognosis is the same as the metacarpal type. CDP brachytelephalangic type is similar, both clinically and radiologically, to warfarin embryopathy and congenital deficiency of multiple vitamin $\mathrm{K}$ dependent coagulation factors. ${ }^{1}$

Costa $e t$ al ${ }^{13}$ presented two unrelated infants with CDP whose mothers had SLE at the 1st International Bone Dysplasia Society in 1993. The first one was a premature (29 weeks) female infant whose mother was treated with prednisone throughout the pregnancy and was positive for anti-La and anti-Ro antibodies. Her upper femoral and tarsal stippling and the typical rash of neonatal lupus subsequently resolved. She was developmentally appropriate at 1 year of age. The second case was male, born at 36 weeks' gestation, and noted to have CDP during investigations for a severe static encephalopathy characterised by hypotonia and multiple contractures. Radiographs in the newborn period had shown coronal clefting of the cervical and upper thoracic spine and stippling in the shoulder and hip areas. These were still present at 6 months of age. The karyotype and levels of very long chain fatty acids were normal. He died at 6 months of age. His mother was treated during the pregnancy with verapamil and prednisone and had taken azathioprine before conception. The type of CDP was not identified.

In 1994 Mansour et $a l^{11}$ reported a premature male child with brachytelephalangic CDP, the second of dizygotic twins delivered spontaneously at 25 weeks' gestation following an otherwise uneventful and drug free pregnancy. The twin sister was normal. A postpartum diagnosis of SLE was made in the West Indian mother whose own mother had died of SLE at 45 years of age. Despite a very stormy neonatal course this infant survived and was developmentally normal by 15 months of age. Several anomalies were noted at birth. There was marked nasal hypoplasia with a depressed nasal bridge, anteverted nares with bilateral alar grooves, and bilateral proptosis. All fingers showed brachydactyly with drumstick swellings of the distal phalanges. Limbs were normal in length and not asymmetrical. The radiological findings were reported as showing epiphyseal stippling of the lumbosacral spine, sagittal clefts of the vertebral bodies, tiny distal phalanges of all fingers, and short second metacarpals with stippling of their proximal ends. The fact that the twin sister was normal suggests that CDP in the sib was probably not related to any direct environmental or teratogenic effect, in keeping with an $\mathrm{X}$ linked recessive condition. At the age of $41 / 2$ years, the right proximal 2nd metacarpal metaphysis was irregular with a large pseudoepiphysis at the site of the previous stippling. The distal phalanges of the thumbs were normal but the others were all extremely short with relatively large epiphyses consistent with CDP brachytelephalangic type (S Mansour, personal communication). 
The findings in our cases together with the three other cases are presented in table 1.

Our cases showed significant long bone shortening, together with metacarpal and phalangeal shortening, consistent with the metacarpal type of CDP. However, in addition, they had moderately severe spinal involvement, stippling of the upper femora, and were both male, more in keeping with CDP brachytelephalangic type. The sagittal vertebral body clefting seen in our case 1 was also present in the case of Mansour et al. ${ }^{11}$

The mothers of our two cases and those of Costa $e t a l^{13}$ and Mansour et $a l^{11}$ all had SLE, although one was diagnosed postpartum. ${ }^{11}$ Prednisone was the only common drug in three of the four mothers. Although animal studies have suggested an increased risk of oral clefts associated with glucocorticoids, several human studies have failed to show teratogenic or toxic effects. Corticosteroids have been used extensively and safely in pregnant patients with SLE and rheumatoid arthritis; there have been no reports of congenital malformations in exposed infants. ${ }^{14}$

Pregnancies in women with SLE are recognised to result in excessive fetal morbidity and mortality. The main complication is congenital heart block which may be identified between 16 and 24 weeks of gestation and other neonatal abnormalities affecting the skin, liver, and blood elements have also been reported to be associated with anti-SSA/Ro and anti-SSB/La antibodies in the maternal and fetal circulation. Neither the incidence nor the severity of neonatal autoimmune disease correlates with maternal disease activity or classification or neonatal antibody titres. ${ }^{15}$

SLE is a multifactorial disease requiring both genetic and environmental factors for disease expression; $57 \%$ of monozygotic twins are concordant for SLE. All forms of drug induced lupus have been shown to be correlated with the presence of the slow acetylator phenotype and subjects who are both slow acetylators and positive for HLA-DR4 have a significant risk of developing hydralazine induced SLE. ${ }^{16}$ The incidence of SLE in the teenage group varies from 4.4 per 100000 white females to 31 per 100000 Oriental females, with 20 per 100000 black females and 13 per 100000 Hispanic females. Racial variation in frequency of SLE may be related to a variety of genetic differences, including differences in oestrogen expression and its impact on the immune system. ${ }^{17}$

Twenty-five percent of SLE patients worsen as a result of pregnancy. Renal disease may first become apparent in patients during the pregnancy, there may be hypertension and toxaemia (as in our case 2), and SLE may greatly worsen or first become apparent during the postpartum period (like the mother from Mansour $\left.e t a l^{11}\right)$. Ninety-five percent of babies born to women with SLE are normal. The various manifestations of lupus in the neonate reflect disease in the mother even though she may be asymptomatic. The risk of clinically evident heart disease is uncertain but much lower than $3 \% .{ }^{16}$ Congenital heart block is currently con- sidered a model of passively acquired autoimmunity, whereby immune abnormalities in the mother lead to the production of autoantibodies (especially SSA/Ro, SSB/La ribonucleoproteins) that cross the placenta and affect the otherwise normally developing fetus. ${ }^{15}$

SLE is an uncommon disorder and both CDP metacarpal type and CDP brachytelephalangic type are extremely rare, only isolated cases or small series having been reported. It is likely, however, that they are both under-reported in view of their relatively mild expressions and recent delineation.

The likelihood of both maternal SLE and neonatal CDP of either metacarpal or brachytelephalangic type occurring by chance in four families with five affected children is remote, suggesting that there could be a direct relationship between maternal SLE and infants with CDP, although this may be no more than genetic susceptibility. Prenatal diagnosis from detailed fetal anomaly ultrasound scanning for both CDP and congenital heart block may be offered to pregnant women with SLE or with a family history of SLE. Prospective studies of patients with SLE will further elucidate the type of CDP, its incidence and precise aetiology.

The authors express their thanks to Dr Caroline A Berry (Genetics, Guy's Hospital) and Dr D K MacIver (Radiology, Lewisham Hospital) for referral of the patients, to Dr Charles Keen (Pathology, Lewisham Hospital) for the postmortem investigations, and to Dr Sahar Mansour (Genetics, St George's Hospital) for the opportunity to review the radiographs of her case.

1 Poznanski AK. Punctate epiphyses: a radiological sign not a disease. Pediatr Radiol 1994;24:418-24.

2 Leicher-Duber A, Schumacher R, Spranger J. Symptomatic calcification in the newborn. Phenocopies of chondrodysplasia punctata. Rofo Fortschr Geb Rontgenstr Neuen Bildgeb Verfahr 1990;152:463-8.

3 Howe AM, Lipson AH, Sheffield LJ, et al. Prenatal exposure to phenytoin, facial development, and possible role for vitamin K. Am f Med Genet 1995;58:238-44.

4 Menger H, Lin AE, Toriello HV, et al. Vitamin K deficiency embryopathy: a phenocopy of the warfarin embryopathy due to a disorder of embryonic vitamin K metabolism. $A m$ $\mathcal{F}$ Med Genet 1997;72:129-34.

5 Ritter M, Menger H, Spranger J. Chondrodysplasia punctata, tibia-metacarpal (MT) type. Am $\mathcal{F}$ Med Genet punctata, tibia-

6 Argo KM, Toriello HV, Jelsema RD, et al. Prenatal findings in chondrodysplasia punctata, tibia-metacarpal type. Ultrain chondrodysplasia punctata, tibia-
sound Obstet Gynecol 1996;8:350-4

7 Burck U. Mesomelic dysplasia with punctate epiphyseal calcifications-new entity of chondrodysplasia punctata. Eur f Pediatr 1982;138:67.

8 Borochowitz Z. Generalized chondrodysplasia punctata with shortness of humeri and brachymetacarpy: humerometacarpal (HM) type: variation or heterogeneity? $A m \mathcal{F}$ Med Genet 1991;41:417.

9 Maroteaux P. Brachytelephalangic chondrodysplasia punctata: a possible X-linked recessive form. Hum Genet 1989;82:167-70.

10 Bennett CP, Berry AC, Maxwell DJ, Seller MJ. Chondrodysplasia punctata: another possible $\mathrm{X}$-linked recessive case. Am f Med Genet 1992;44:795-9.

11 Mansour S, Liberman D, Young I. Brachytelephalangic chondrodysplasia punctata in an extremely premature chondrodysplasia punctata in an extre
infant. Am $\mathcal{F}$ Med Genet 1994;53:81-2.

12 Taybi H, Lachman RS, eds. Radiology of syndromes, metabolic disorders, and skeletal dysplasias. 4th ed. St Louis: Mosby, 1996:776-86.

13 Costa T, Tiller G, Chitayat D, Silverman E. Maternal systemic lupus erythematosis (SLE) and chondrodysplasia punctata in two infants. Coincidence or association? 1 st Meeting of Bone Dysplasia Society, Chicago, fune 1993.

14 Ramsey-Goldman R, Schilling E. Immunosuppressive drug use during pregnancy. Rheum Dis Clin North Am 1997;23 149-67.

15 Tseng CE, Buyon JP. Neonatal lupus syndromes. Rheum Dis Clin North Am 1997;23:31-54.

16 Jacobs JC. Systemic lupus erythematosus. In: Pediatric rheumatology for the practitioner. 2nd ed. Berlin: Springer-Verlag, 1992:409-520

17 Lehman TJA. A practical guide to systemic lupus erythematosus. Pediatr Clin North Am 1995;42:1223-37. 